\title{
Fluorosis dental: Prevalencia, grados de severidad y factores de riesgo en niños de 7 a 13 años del Cantón Cuenca
}

\author{
Janeth Parra C. ${ }^{1}$, Diana Astudillo N. ${ }^{2}$, Norma Cedillo A. ${ }^{2}$, Galo Ordoñez E. ${ }^{3}$, Fernando \\ Sempértegui ${ }^{4}$
}

${ }^{1}$ Facultad de Odontología, Universidad de Cuenca, Av. 12 de Abril s/n, Cuenca, Ecuador

${ }^{2}$ Facultad de Ciencias Químicas, Universidad de Cuenca, Av. 12 de Abril s/n, Cuenca, Ecuador

${ }^{3}$ Facultad de Ingeniería, Universidad de Cuenca, Av. 12 de Abril s/n, Cuenca, Ecuador

${ }^{4}$ Universidad Central, Facultad de Medicina, Corporación Ecuatoriana de Biotecnología, Quito, Ecuador

Autor para la correspondencia: judith.parra@ucuenca.edu.ec

Fecha de recepción: 8 de junio 2012 - Fecha de aceptación: 30 de julio 2012

\section{RESUMEN}

El objetivo de esta investigación fue determinar la prevalencia de fluorosis dental en los niños escolares de áreas urbanas y rurales del Cantón Cuenca, así como potenciales factores de riesgo: flúor en el agua y la sal de consumo. Además, se estudió la asociación de fluorosis con proteína C-reactiva (PCR) y talla. Se realizó un estudio transversal en 222 escolares del área rural y 285 del área urbana, de 7 a 13 años. Se relacionó el grado de fluorosis con la concentración de flúor en el agua y la orina, la sal de consumo y la concentración de PCR en la sangre. La prevalencia de fluorosis dental fue mayor en el área rural que en la urbana: 80,7 versus 74\%. Según el Índice de Fluorosis de Dean, la fluorosis moderada y severa tuvieron porcentajes más elevados en el área rural que en la urbana: de $4,2$ a $23 \%$ versus 0,4 a $4,1 \%$, respectivamente. La edad (OR: 1,23 ; IC: 1,01 a 1,$51 ; p=0,04)$ y el contenido del flúor en la sal (OR: 7,3; IC: 2,29 a 23,4; $p=0,001$ ) se asociaron significativamente con la severidad de la fluorosis solo en los niños del área rural. La severidad de la fluorosis no se asoció con la concentración de PCR ni con la talla de los niños. Este estudio sugiere que los niños de mayor edad de escuelas rurales tienen más riesgo de fluorosis, posiblemente por persistente y excesivo consumo de flúor en la sal.

Palabras clave: Fluorosis dental, edad, talla, flúor en el agua, orina, sal de consumo, proteína Creactiva, área urbana y rural.

\begin{abstract}
The purpose of the investigation was assessment of the prevalence of dental fluorosis in scholar children living in the urban and rural areas of Cuenca Canton and to define the responsible risk factors, such as fluor concentration in drinking water and sodium. Also the relation between fluorosis and C-reactive protein (CRP) and stature were examined. A transversal study was carried out on 222 children living in the rural area and 285 in the urban area, 7 to 13 years old. The survey revealed that the degree of fluorosis is related to the fluor concentration in drinking water and urine, the salt intake and the CRP concentration in blood. The prevalence of dental fluorosis is higher in the rural than the urban area: $80,7 \%$ versus $74 \%$. According to Dean's index of fluorosis, the percentage of moderate and severe fluorosis are considerably higher in the rural than in the urban area: respectively 4,2 and $23 \%$ versus 0,4 and $4,1 \%$. Age (OR: 1,23; IC: 1,01 a 1,$51 ; p=0,04)$ and fluor content in salt (OR: 7,3; IC: 2,29 a 23,$4 ; p=0,001$ ) are significantly related with the severity of fluorosis, but only in the children living in rural areas. The degree of fluorosis is not related with the CRP concentration, neither with the stature of the children. The survey clearly reveals that the older children in the rural
\end{abstract}


areas are more vulnerable to fluorosis, most likely the consequence of the high fluor intake due to the daily excessive salt consumption.

Keywords: Dental fluorosis, age, stature, fluor in drinking water, urine, salt consumption, C-reactive protein, urban and rural area.

\section{INTRODUCCIÓN}

La fluorosis dental es una hipomineralización de la superficie del esmalte del diente que se desarrolla durante la formación del diente, pudiendo presentarse una fluorosis leve en forma de manchas blancas o líneas en la superficie del esmalte; moderada y severa con manchas ásperas e irregularidades ya sea de color blanco o café. La fluorosis no puede presentarse una vez que la formación del esmalte está completa y los dientes han brotado (Harris y García-Godoy, 2005). La fluorosis dental es un marcador de una ingesta de flúor que sobrepasa la dosis óptima, y puede producir diferentes afectaciones en la salud a más de los daños en la mineralización de los huesos (Harrison y col., 1990), podrían estar afectados los sistemas intracelulares anti-oxidantes, lo que explicaría, al menos en parte, los daños óseos y otros descritos en músculo y cerebro (Shivarajashankara y col., 2001; Vani y col., 2001).

La utilización de fluoruros para prevenir la caries dental ha sido ampliamente utilizada desde la década del 40 del siglo pasado cuando se demostró su utilidad. Numerosos países han reportado una reducción en la prevalencia de caries como consecuencia de la incorporación de fluoruros al agua de consumo (Franco y col., 2003; Montero y col., 2007), así como a la utilización de dentífricos fluorados, suplementos de flúor, sal y leche fluorada (Murray y col., 1991; Lennon y Ketley, 2001; Castillo y col., 2011) lo que ha traído como consecuencia una disminución de la prevalencia de caries dental, pero a su vez se ha incrementado la fluorosis dental. Sin embargo, existe mucha controversia científica sobre la efectividad/riesgo del flúor en relación a su toxicidad, ingesta total del flúor, absorción, e impacto en la salud humana. El US Public Health Service estableció un estándar óptimo para el fluoruro en el agua para beber entre 0,7 y 1,2 ppm (Harris y García-Godoy, 2005). El flúor ingerido, se absorbe rápidamente en las vías gastrointestinales y se excreta en las heces. Del fluoruro absorbido en la circulación sanguínea aproximadamente la mitad se fija en los tejidos duros (huesos, y dientes por erupcionar), el resto se elimina por la orina. Cierta exposición a los fluoruros es inevitable en el ambiente humano, su indicador más precoz es una fluorosis dental seguida por la fluorosis esquelética, que puede variar desde una osteoesclerosis asintomática, hasta un crecimiento óseo anormal, además puede presentarse efectos tóxicos en otros órganos como son el riñón, sistema endocrino (Bordoni y Squassi, 1992) . La ingestión excesiva de flúor durante los primeros años de vida puede dañar la estructura del diente dando lugar a un defecto en el esmalte conocido como fluorosis dental. Según Wefel y Donly (1999) el 32\% de los niños de América tienen actualmente alguna forma de fluorosis dental; de los cuales de 2 a $4 \%$ es de grado moderado a severo.

Estudios realizados en México (Hurtado y col., 2005) determinaron que la fluorosis dental, la fluorosis del esqueleto, y las fracturas óseas, son tres riesgos potenciales, como consecuencia de altas ingestas de flúor. La Escuela de Odontología Humboldt-University Berlin, Germany. (Zimmer y col., 2003) y la mayoría de las asociaciones científicas Europeas no recomiendan el uso de flúor en suplementos fluorados para la prevención de la caries, debido a su potencial efecto sistémico perjudicial. Muchos países han elegido la fluoración de la sal para prevenir la caries dental, a niveles comparables a los de la fluoración del agua. El nivel de suplemento de fluoruro de la sal refinada debe ser mínimo de $250 \mathrm{mg} \mathrm{KF} / \mathrm{Kg}$ de sal y $225 \mathrm{mg} \mathrm{NaF} / \mathrm{Kg}$ de sal (Cardenas, 2009); sin embargo, la desventaja estriba en la dificultad para conocer la cantidad de sal ingerida; este criterio se puede apreciar en los resultados obtenidos en un estudio realizado en Colombia (Arrieta y col., 2011) para determinar fluorosis dental y factores de riesgo asociados en doscientos treinta niños, en el cual se obtuvo una prevalencia de fluorosis dental alta de $65 \%$ y se concluyó que los factores que explican la presencia de fluorosis dental fueron: la cantidad de sal fluorada que se usa en la preparación de los alimentos y la primera aplicación de flúor antes de los 4 años. Cuando son varias las fuentes de ingestión de fluoruros se incrementa la cantidad total de flúor sistémico y lejos de prevenir la caries, 
producen un potencial daño para la salud al producir fluorosis dental. De ahí la importancia de establecer un sistema de vigilancia sobre los principales elementos que pueden aportar flúor al organismo humano (Rosales y Maritza, 2004).

En 1997 el Ministerio de Salud Pública y la Dirección de Estomatología del Ecuador pusieron en marcha el Programa Nacional de Fluoración de la Sal. En los estudios basales previos al inicio de dicho programa, se determinó que en Ecuador la caries dental y la enfermedad periodontal constituyen un problema de salud pública. Dichas patologías tienen una alta prevalencia para cada una: $88,2 \%$ y 87\%. En cuanto a la fluorosis dental, el porcentaje nacional de escolares afectados fue del 9,2\%, habiendo dentro de este porcentaje una fluorosis dudosa del 4,7\%. Según estos estudios el flúor en el agua de la provincia del Azuay es bajo, entre 0 a $0,39 \mathrm{ppm}$, e igual el contenido del flúor en la orina. De acuerdo a lo resultados obtenidos se determina que el monitoreo del flúor en la orina es un método válido para la vigilancia del programa de fluoración de la sal de consumo humano en el país (Ministerio de Salud Pública del Ecuador/Organización Panamericana de Salud (OPS), 1997a-d). Existiendo otros estudios realizados en México y Chile que corroboran este concepto (Guerrero y col., 1996; Muzzo y Villa, 2001). Sobre la base de diferentes estudios el Ministerio de Salud Pública y la Dirección de Estomatología del Ecuador recomendaron: (a) un monitoreo químico de la concentración de flúor en el agua al menos una vez por año; (b) la realización de estudios periódicos sobre fluorosis dental; y (c) de no prescribir el uso de suplementos fluorados, así como vigilar el cepillado dental en niños menores de cinco años.

Con estos antecedentes se efectuó un estudio que tuvo como objetivo determinar el grado de fluorosis dental, su relación con la concentración de flúor en el agua y sus efectos tisulares (hueso) y metabólicos en niños escolares de las parroquias rurales y urbanas del Cantón Cuenca. Los objetivos específicos fueron: (a) determinar la prevalencia de la fluorosis dentaria y su severidad en niños de 7 a 13 de edad; (b) valorar la concentración de flúor en las fuentes de agua del Cantón Cuenca y establecer la carga de flúor diaria mediante la determinación del flúor en la orina; (c) determinar el aporte de flúor para la carga diaria mediante la cuantificación del flúor en la sal de consumo en niños con fluorosis moderada/severa y en controles sin fluorosis; (d) establecer la presencia o no de inflamación mediante la medición de la proteína C-reactiva (PCR); (e) evaluar si existe alteración en el crecimiento en niños con fluorosis y sin fluorosis, e identificar factores de riesgo asociados a la fluorosis.

\section{MATERIALES Y MÉTODOS}

\subsection{Materiales}

Se trata de un estudio epidemiológico transversal, en el cual se estableció la prevalencia y el grado de fluorosis dentaria y las condiciones de salud buco dental en una muestra de niños escolares del Cantón Cuenca, que se ha conformado por 19 parroquias rurales (169 planteles escolares) y 15 parroquias urbanas (149 planteles escolares). Luego, en un estudio de casos y controles, se comparó el aporte de flúor sistémico (sal) y los mecanismos intracelulares anti-oxidantes en niños con fluorosis moderada y severa versus niños sin fluorosis. La muestra representativa correspondió a 294 escolares de las parroquias rurales y urbanas. El cálculo de la muestra se realizó con el programa EPIDAT 3.1, siguiendo los siguientes parámetros: tamaño de la población rural 7.240 y urbana 10.000; nivel de confianza 95\%; proporción de fluorosis esperada 15\%; y precisión absoluta de 1 a $4 \%$. Se excluyeron los niños con historia de enfermedades renales, niños que padezcan infección actual, niños que estén bajo tratamiento médico o tomando medicamentos. Los niños fueron incluidos con el consentimiento firmado de sus padres. Se retiraron del estudio 72 escolares rurales por cambio inesperado de escuela y de domicilio y 9 escolares urbanos que no aceptaron continuar en el estudio, quedando la muestra constituida por 222 y 285 escolares respectivamente. 


\subsection{Metodología}

Para el examen buco-dental de los escolares se utilizó las fichas clínicas individuales con su odontograma respectivo. Se estandarizó a los examinadores de acuerdo a los criterios de la OMS. Para el examen se utilizó un espejo \#5 plano y explorador; con luz natural, previa la remoción de placa dento-bacteriana, se secaron las piezas dentarias con algodón y se registró el índice de fluorosis dental. El Índice de Dean modificado, se obtiene mediante el examen de las caras vestibulares de los dientes: $11,12,13,21,22$ y 23, de cada individuo, para lo cual se utilizó una escala con peso numérico que va del 0 al 4 . Así: $0=$ normal; $1=$ muy leve $(<1 / 3) ; 2=$ leve $(=2 / 3) ; 3=$ moderado $(>2 / 3) ; 4=$ severo (la superficie dentaria está muy afectada, el diente se presenta corroído) (Dean, 1934; WHO, 1997; Harris, 2005). Los criterios para definir cada valor son los siguientes:

- Normal a dudoso: la superficie del esmalte es lisa, brillosa, generalmente de color blanco cremoso.

- Muy leve: el esmalte contiene pequeñas zonas opacas de color blanco papel, dispersas irregularmente en el diente, pero que afectan al menos al $25 \%$ de la superficie dentaria. Se observa no más de 1 a $2 \mathrm{~mm}$ de opacidad blanca, en el extremo de cúspide de caninos, premolares y molares.

- Leve: La opacidad del esmalte es mayor que la correspondiente al código 1, pero abarca menos del $50 \%$ de la superficie del esmalte.

- Moderado: El 50\% o más de la superficie del diente está afectado por la fluorosis. Las superficies del esmalte pueden aparecer desgastadas.

- Severo: la superficie del esmalte está muy afectada y la hipoplasia es tan marcada que puede afectar a la forma general del diente presentando un aspecto corroído; es un esmalte con excavaciones separadas y confluentes.

El grado de fluorosis dental de cada niño se obtuvo de acuerdo a la valoración de la penúltima lesión más grave con relación a los criterios de Dean, los mismos que se ordenaron de menor a mayor gravedad. La prevalencia de la fluorosis dental se obtuvo en relación a la presencia de fluorosis dental y de acuerdo al nivel de afectación. Para los análisis de regresión logística se agrupó el Índice de Dean en dos grupos: 1:(sano-muy leve-leve) 2: (moderada-severo).

En este estudio no se consideró el grado de dudoso; además, se examinó y se registró los valores correspondientes de las caras vestibulares de todas las piezas permanentes presentes. Mediante una encuesta se estableció las condiciones de salud buco-dental de los niños, y se registró la talla de los escolares mediante un procedimiento estandarizado (doble medición). Además, se diseñaron utilizando un sistema de posicionamiento global (GPS) la cartografía geográfica de las escuelas urbanas y rurales y de sus fuentes de agua. Se obtuvo 80 muestras de agua. El análisis químico del agua se realizó con el método de potenciómetro: se utilizó un pH-metro de mesa marca ORION 3STAR código \#1112000, con un electrodo ORION Flúor Ion código \#9609 BNW (Aguilar, 2001). Para la determinación del flúor en la orina se realizó con la técnica del electrodo de ion específico (Ministerio de Salud-UNICEF, 2000). Para evaluar el contenido de flúor en el agua y en la orina se tomó en consideración los siguientes rangos: de 0,0 a $0,39=$ bajo; de 0,4 a $0,69=$ medio; de 0,7 a 1,49 = óptimo; y de 1,5 y más = alto. En la muestra de orina la exposición alta corresponde a valores $\geq$ $1,50 \mathrm{ppm}$ F/gramo de creatinina y la exposición baja a valores entre 0,0 a 1,49 ppm F/gramo de creatinina.

En niños con fluorosis moderada o severa y en niños con control sin fluorosis se midió el consumo de flúor en la sal de la dieta de un día al azar. En la visita al domicilio se solicitó una muestra de la sal disponible en el momento de la preparación del almuerzo y se cuantificó la cantidad usada. De igual manera se procedió en las escuelas que tienen programa de almuerzo escolar, a fin de estimar el aporte de sal en la dieta, se pesó la ración de la comida principal (almuerzo) asignada al niño y se efectuaron los cálculos correspondientes. En escolares con fluorosis moderada y severa y en los controles (sin fluorosis) se realizó un examen de sangre $(5 \mathrm{ml})$ para determinar los marcadores PCR. La sangre se obtuvo por punción venosa en la mañana con el niño en ayunas de 12 horas.

Los datos se recolectaron en el formulario diseñado para el efecto y luego de revisados y corregidos se transfirieron a una base de datos del programa SPSS versión 15. Se calculó la 
prevalencia (\%) de fluorosis en la población general, por área urbano/rural, por edad y según el Índice de Dean. Se calculó el promedio y el desvío estándar (SD) de la concentración de flúor en orina en la población escolar, y la concentración de flúor en agua por parroquia. Se estimó el riesgo entre fluorosis dental y posibles factores asociados mediante tablas de contingencia y pruebas de chicuadrado, $\chi^{2}$ ). Se realizaron modelos de regresión logística múltiple para examinar la relación entre fluorosis y flúor en el agua; flúor en sal (porción de comida), flúor en la orina, y marcador PCR. Los modelos fueron controlados por edad y género.

\section{RESULTADOS}

Las características generales de los dos grupos escolares que participaron en el estudio se muestran en la Tabla 1. Los niños tuvieron una edad mínima de 7 años y máxima de 13. Las variables independientes examinadas entre los dos grupos de escolares son edad, rango de edad, sexo, talla, y talla por rango de edad. Según la prueba de diferencia de promedios y porcentajes $\left(\chi^{2}\right)$ entre los dos grupos la diferencia no fue estadísticamente significativa $(p \geq 0,05)$, es decir los dos grupos de estudio son similares en cuanto a estas variables. La concentración de flúor en el agua en las parroquias urbanas $(0,135 \pm 0,057 \mathrm{ppm})$ es más elevado que en las parroquias rurales $(0,00240 \pm 0,0055 \mathrm{ppm})$, con la diferencia de promedios estadísticamente significativa $(\mathrm{p}=0,000)$. Sin embargo, la concentración de flúor en el agua en los grupos de estudio es bajo en relación a la dosis óptima.

Tabla 1. Características de los escolares de 7 a 13 años de las parroquias rurales y urbanas del Cantón Cuenca.

\begin{tabular}{|c|c|c|c|c|c|}
\hline \multirow{2}{*}{ Variable } & & \multicolumn{2}{|c|}{ Área } & \multirow{2}{*}{\multicolumn{2}{|c|}{$\mathrm{p}$}} \\
\hline & & Rural & Urbana & & \\
\hline Edad (años) & $(\overline{\mathrm{y}} \pm \mathrm{DS})$ & $9,60 \pm 1,54$ & $9,41 \pm 1,53$ & 0,17 & NS \\
\hline Edad (7-10 años) & $\%$ & 68,9 & 71,6 & 0,51 & NS \\
\hline Edad (11-13 años) & $\%$ & 31,1 & 28,4 & 0,59 & NS \\
\hline Género femenino & $\%$ & 45,0 & 53,3 & 0,64 & NS \\
\hline Talla $(\mathrm{cm})$ & $(\overline{\mathrm{y}} \pm \mathrm{DS})$ & $129,0 \pm 9,35$ & $130,0 \pm 9,35$ & 0,64 & NS \\
\hline Talla (cm) (7-10 años) & $(\overline{\mathrm{y}} \pm \mathrm{DS})$ & $125,1 \pm 7,99$ & $126,8 \pm 7,92$ & 0,90 & NS \\
\hline Talla (cm) (11-13 años) & $(\overline{\mathrm{y}} \pm \mathrm{DS})$ & $136,2 \pm 7,55$ & $139,4 \pm 7,17$ & 0,60 & NS \\
\hline Flúor en el agua (ppm) & $(\overline{\mathrm{y}} \pm \mathrm{DS})$ & $0,135 \pm 0,057$ & $0,002 \pm 0,005$ & 0,00 & $S$ \\
\hline Número de escolares & & 222 & 285 & & \\
\hline
\end{tabular}

* Los promedios del flúor en el agua en el área rural y urbana son bajos en relación a la dosis óptima $=0,7$ a 1,2 ppm.

El Tabla 2 presenta la prevalencia de fluorosis dental, sus grados de severidad según el Índice de Dean y el contenido de flúor en la orina de los dos grupos de escolares que participaron en el estudio. La prevalencia de fluorosis dental en las parroquias rurales del Cantón Cuenca es de 80,7\% y en las urbanas es de $74 \%$, al obtener la diferencia de porcentajes $\left(\chi^{2}\right)$ entre los dos grupos la diferencia no fue estadísticamente significativa $(\mathrm{p} \geq 0,06)$. En cuanto a los grados de severidad de acuerdo al Índice de Dean los resultados fueron los siguientes: el porcentaje del grado de fluorosis severo de los escolares rurales es $4,1 \%$ y la fluorosis severa de los escolares urbanos es $0.4 \%$. La diferencia de porcentajes fue estadísticamente significativa $(\mathrm{p} \leq 0,008)$. El grado de fluorosis moderado entre los escolares rurales fue de $23 \%$ en relación al de los escolares urbanos $4,1 \%$; la diferencia fue estadísticamente significativa $(\mathrm{p} \leq 0,000)$. El grado de fluorosis muy leve se presentó en un porcentaje mayor en los escolares urbanos $45,6 \%$ versus $23,4 \%$ al de los rurales, con una diferencia estadísticamente significativa $(\mathrm{p} \leq 0,000)$. La diferencia entre los grados leve y sano en los dos grupos 
de escolares no fueron estadísticamente significativos $(\mathrm{p}=0,10$ y $\mathrm{p}=0,08)$ respectivamente. En cuanto al flúor en la orina, el grupo de escolares rurales presentó mayor contenido de flúor: 1,5 $\pm 0,58$ ppm en relación al grupo urbano $0,83 \pm 0,49 \mathrm{ppm}$. La diferencia de promedios demostró ser estadísticamente significativa $(\mathrm{p}=0,02)$.

Tabla 2. Prevalencia de fluorosis, grados de severidad y concentración de flúor en la orina de los niños de 7 a 13 años de las áreas urbanas y rurales del Cantón Cuenca.

\begin{tabular}{|c|c|c|c|c|c|}
\hline \multirow{2}{*}{ Variable } & & \multicolumn{2}{|c|}{ Área } & \multirow{2}{*}{\multicolumn{2}{|c|}{$\mathrm{p}$}} \\
\hline & & \multirow{3}{*}{$\begin{array}{l}\text { Rural } \\
80,7\end{array}$} & \multirow{3}{*}{$\begin{array}{l}\text { Urbana } \\
74,0\end{array}$} & & \\
\hline Fluorosis & $\%$ & & & 0,06 & NS \\
\hline $\begin{array}{l}\text { (muy leve-leve- } \\
\text { moderado-severo) }\end{array}$ & & & & & \\
\hline Severo & $\%$ & 4,1 & 0,4 & 0,008 & $\mathrm{~S}$ \\
\hline Moderado & $\%$ & 23,0 & 4,2 & 0,000 & $S$ \\
\hline Leve & $\%$ & 23,4 & 45,6 & 0,000 & $S$ \\
\hline Muy leve & $\%$ & 30,2 & 23,5 & 0,10 & NS \\
\hline Normal a dudoso & $\%$ & 19,4 & 26,3 & 0,08 & NS \\
\hline Flúor en la orina $(\mathrm{mg})$ & $(\overline{\mathrm{y}} \pm \mathrm{DS})$ & $1,5 \pm 0,58$ & $0,83 \pm 0,49$ & 0,02 & $S$ \\
\hline Número de escolares & & 222 & 285 & & \\
\hline
\end{tabular}

* Fluorosis dental según la escala de Dean (normal a dudoso, muy leve, leve, moderada, severo)

Mediante una encuesta se estableció la condición de salud buco-dental de los niños, se logró registrar a 222 niños rurales y se obtuvieron los siguientes resultados. El 57,2\% se cepilla los dientes en la mañana; antes de acostarse lo realiza el 53,4\%. El 91\% usa pasta dental; el $9 \%$ usa varias sustancias con jabón, bicarbonato, entre otras. La cantidad de pasta que usan es: cepillo completo el 19,9\%; mitad de cepillo el 41,2\%; tercio de cepillo el 30,8\% y el 8,1\% no contesta la pregunta. En cuanto al antecedente de haber recibido o no la recomendación sobre el uso de suplementos fluorados, el $5,4 \%$ responden que si, el $87,3 \%$ responden que no y $7,3 \%$ no responde la pregunta. Los suplementos que toman son: el Zima flúor lo utiliza el 0,5\%; Poly-Vi-Flúor 1,8\%; chicle con flúor $0,9 \% ; 6,3 \%$ no recuerda el nombre del suplemento; y el 90,5\% no contesta a la pregunta. La sal de consumo diario es: Crisal 72,4\%; Pacífico 2,7\%; y el 24,9\% no contesta la pregunta. Los resultados de los escolares urbanos, 285 escolares en total, fueron los siguientes: el 82,1\% se cepilla los dientes en la mañana; antes de acostarse lo realiza el $72 \%$. El cepillado lo efectúan con pasta dental es $97 \%$; y el $3 \%$ de los escolares usa varias sustancias con jabón, bicarbonato y otras. La cantidad de pasta que utilizan es la siguiente: cepillo completo el $28 \%$, mitad de cepillo un $46 \%$, tercio de cepillo un $17,8 \%$, el $8,2 \%$ no contesta la pregunta. En cuanto al antecedente de haber recibido o no la recomendación sobre el uso de suplementos fluorados, el 11,3\% responden que si, $86,5 \%$ responden que no; $2,2 \%$ no responde la pregunta. Los suplementos que toman son el Zima flúor lo utiliza el 1,1\%; Poly-Vi-Flúor el 2,9\%; chicle con flúor el 1,1\%; el 13,8\% no recuerda el nombre del suplemento; y el $81,1 \%$ no contesta la pregunta. La marca de sal de consumo diario es la Crisal 71,6\%; el 28,4\% no contesta la pregunta.

Tabla 3. Asociación entre edad, flúor en la sal de la comida y área de residencia con fluorosis dental.

\begin{tabular}{cccc}
\hline Variable & OR* & IC* $(95 \%)$ & $\mathrm{p}^{*}$ \\
\hline Edad (años) & 1,23 & 1,01 a 1,51 & 0,040 \\
Flúor en la sal de comida $(\mathrm{mg})$ & 7,30 & 2,29 a 23,4 & 0,001 \\
Área rural & 3,90 & 1,20 a 7,50 & 0,010 \\
\hline
\end{tabular}

*OR $=$ odds ratio, $\mathrm{IC}=$ intervalo de confianza; $\mathrm{p}=$ significación estadística 
En niños con fluorosis moderada o intensa y en niños sin fluorosis de los escolares rurales y urbanos se midió el consumo de flúor en la sal de la dieta de un día al azar. Además se complementó el análisis de fluorosis con un examen de sangre $(5 \mathrm{ml})$ para determinar los marcadores PCR. Se efectuó un análisis de regresión múltiple y para obtener el modelo se introdujeron las variables independientes: edad, género, flúor en la sal promedio que ingieren los niños en su plato de comida diario, el marcador PCR y flúor en la orina. Se eliminaron una a una las variables menos significativas: PCR; flúor en la orina hasta que se obtuvo un modelo reducido y se concluye que en el modelo final quedan las variables edad y flúor en la sal de la dieta, ya que dichas variables tienen valores de significación bajos.

\section{DISCUSIÓN}

En el Ecuador en el año 1997 el Ministerio de Salud Pública y la Dirección de Estomatología en sus estudios basales previos al inicio del Programa de fluoración de la sal, determinaron que la prevalencia en el Ecuador de la fluorosis dental, utilizando el Índice de Dean, fue del 9,2\%, habiendo dentro de este porcentaje una fluorosis dudosa del 4,7\% (Ruiz y col., 1997). Los resultados obtenidos en el presente estudio demuestran que la fluorosis dental en los escolares de las parroquias rurales del Cantón Cuenca ha aumentado considerablemente (80,7\%), también en los escolares urbanos (74\%). Si bien en los escolares rurales los porcentajes mayores de fluorosis se encuentran en el nivel leve $(30,2 \%)$ y muy leve $(23,4 \%)$; sin embargo existen porcentajes significativos de grado moderado $(23 \%)$ e intensa $(4,1 \%)$, lo que debe constituirse en motivo de preocupación en el área de salud pública. En los escolares urbanos el problema es menor, ya que la fluorosis muy leve es 45,6\% y la leve $23,5 \%$; y en porcentajes moderada $4,2 \%$ y severa $0,4 \%$. El flúor en el agua en todas las parroquias rurales y urbanas del Cantón Cuenca tiene una concentración entre 0,0 y 0,39 ppm, que corresponde a un nivel bajo, que coincide con los datos obtenidos en 1997 por el Ministerio de Salud Pública y la Dirección de Estomatología (Ruiz y col., 1997).

El presente estudio concuerda con los resultados de un estudio de prevalencia de fluorosis dental realizado en la ciudad de Medellín, Colombia, en 1330 niños de 6 a 13 años, cuyos resultados fueron del $81 \%$ (Ramírez y col., 2006). En dicho estudio el 72,3\% tenían grados leves de fluorosis dental (leve y moderada), y un $8,8 \%$ severo. En nuestro estudio, en los escolares rurales el grado leve de fluorosis fue del 30,2\%, muy leve 23,45\%; y el grado moderado y severo $23 \%$ y $4,1 \%$ respectivamente. Quizás esta diferencia se deba a que el estudio realizado en Colombia se utilizó el Índice de Thylstrup y Fejerskov (Fejerskov y col., 1988) que da mayor énfasis a los grados moderado y severo y en el nuestro se utilizó el Índice de Dean. Sin embargo a pesar de que existe limitación en el cotejo estricto de los resultados de los dos estudios, los resultados en la prevalencia son bastantes equivalentes.

\section{CONCLUSIONES}

En este estudio se demuestra que existe una alta prevalencia de fluorosis dental en los niños de 7 a 13 años de las parroquias rurales y urbanas del Cantón Cuenca. De acuerdo al grado de severidad, para la fluorosis muy leve y leve se obtiene un porcentaje del 53,6\% en los escolares rurales; en cambio en los escolares urbanos este porcentaje es mayor, igual a 69,1\%. En los escolares rurales el $23 \%$ tiene fluorosis moderada, porcentaje mayor en relación con los urbanos 4,2\%. De igual manera sucede con respecto a la fluorosis severa donde los porcentajes corresponden al $4,1 \%$ y al $0,4 \%$ respectivamente. Los resultados obtenidos demuestran que los niños de las parroquias rurales son los más afectados; aunque también la fluorosis dental es un problema importante entre los niños de procedencia urbana. En cuanto al contenido de flúor en el agua, los hallazgos corresponden a un nivel bajo de 0,0 a 0,39 
ppm. El bajo nivel indica que el flúor en el agua no constituye un factor de riesgo para la fluorosis dental.

Al realizar el análisis de regresión logística del Índice de Dean en relación al género, edad, proteína PCR, flúor en la orina, y el flúor en la sal promedio que ingieren los niños en su plato de comida diario, se determina que en los escolares rurales, mientras más tiempo el niño ha estado expuesto al flúor, la fluorosis aumenta en 1,23 veces. El consumo de sal tiene impacto en la fluorosis dental, es decir que por cada miligramo de flúor que se añade a la sal de la comida de los niños, el riesgo de fluorosis aumenta en 7,3 veces. En los escolares urbanos estas variables no constituyen factores de riesgo.

Es necesario realizar nuevos estudios para identificar otras fuentes de ingesta sistémica de flúor y de determinar los factores de riesgo en la población. Debe considerarse que el hábito de ingerir sal con las frutas, como una expresión del gusto o hábito es un factor cultural que favorece la presencia de la fluorosis dental; además, se recomienda realizar un estudio de los hábitos dietéticos de los escolares de esta población y realizar talleres de educación de salud buco-dental en los escolares de parroquias urbanas y rurales. Por último, se debe considerar la posibilidad de ajustar la carga de flúor en la sal de consumo diario según las prácticas dietéticas locales para evitar una excesiva carga de flúor que podría estar asociada a la fluorosis dental.

\section{AGRADECIMIENTOS}

Agradecemos a la Universidad de Cuenca y a la Dirección de Investigación (DIUC) por el financiamiento otorgado para la ejecución de esta investigación; a la Corporación Ecuatoriana de Biotecnología en Quito por su aporte con los análisis de sangre; al Dr. Carlos Flores por su asesoramiento estadístico y sobre todo a los niños y docentes de las escuelas rurales y urbanas del Cantón Cuenca por la colaboración en el estudio.

\section{BIBLIOGRAFÍA}

Aguilar, P., 2001. Validación del método potenciométrico por Ión Selectivo para la determinación de Flúor en sal, agua y orina. Rev. Peruana Med. Exp. Salud Pública, 18(1-2), 21-23.

Arrieta, K., Gonzalez, F., Luna, R., 2011. Exploring the risk of dental fluorosis in children at the Universidad of Cartagena's Odontology Clinics. Rev. Salud Pública, 13(4), 672-683.

Bordoni, N., Squassi, A., 1992. Odontología preventiva. Módulo 3: Medidas y programas preventivos. Organización Panamericana de la Salud (OPS), p. 8-10.

Cardenas, J.D., 2009. Fundamentos de odontología: Odontología pediátrica. $4^{\text {a }}$ edición. Quebecor Word, Bogotá S.A., Colombia, p. 179-183.

Castillo, R., Perona, G., Kanashiro C., Perea M., Silva-Esteves, S., 2011. Estomatología pediátrica.1 ${ }^{\text {a }}$ edición. Editorial Ripano, Péru, p. 115-120.

Dean, H.T., 1934. Classification of mottled enamel diagnosis. J. Amer. Dental Assoc., 21, 1421-1426.

Fejerskov, O., Firoze, M., Baelum, V., Moller, I., 1988. Dental Fluorosis: A handbook for health workers. Munksgaard, Copenhagen.

Franco, A.M., Martignon, S., Saldarriega, A., González, M.C., Luna, L., Ocampo, A., Villa, A., Arbeláez, M.I., 2003. Ingesta de flúor en niños de 2 a 4 años en cuatro ciudades Colombianas. Rev. de la Asociación Colombiana de Facultades de Odontología (ACFO), Medellín, Colombia, p. 10-11.

Guerrero, J., Velásquez, I., Ledesma, C., 1996. Concentración de flúor en la orina en niños radicados en la ciudad de México. Rev. Mexicana de Pediatría, 65, 236-241. 
Harris, N.O., García-Godoy, F., 2005. Odontología preventiva primaria. $6^{\mathrm{a}}$ edición en inglés, $2^{\mathrm{a}}$ edición español. Editorial Manual Moderno, México, p. 144-145 y 147-150.

Harrison, J.E., Hitchman, A.J., Haltrope, M.E., 1990. The effects of fluoride on ectopic bone formation. J. Bone Miner. Res., 5(1), 81-85.

Hurtado-Jiménez, R., Gardea-Torresdey, J., 2005. Estimation of exposure to fluoride in Los Altos de Jalisco México. Rev. Salud Públ. México, 47(1), 58-63.

Lennon, M.A., Ketley, C.E., 2001. Determination of fluoride intake from urinary fluoride excretion data in children drinking fluoridated school milk. Caries Res., 35, 252-257.

Ministerio de Salud Pública del Ecuador/OPS, 1997a. Programa de fluoración de la sal de consumo humano. Dirección Nacional de Estomatología, Quito, Ecuador.

Ministerio de Salud Pública del Ecuador/OPS, 1997b. Estudio de excreción de fluoruro en la orina en niños de 3 a 5 años, en tres zonas de Ecuador. Dirección Nacional de Estomatología, Quito, Ecuador.

Ruiz y colb. 1997. Estudio epidemiológico de salud bucal en escolares fiscales. Menores de 15 años del Ecuador. Dirección Nacional de Estomatología, Quito, Ecuador.

Ruiz y colb. 1997. Estudio del contenido natural de flúor en el agua de consumo humano de los abastecimientos en Escolares. Dirección Nacional de Estomatología, Quito, Ecuador, p. 7-10.

Ministerio de Salud-UNICEF, 2000. Área de vigilancia nutricional desarrollo de comunidades centinela sobre alimentación y nutrición: micronutrientes convenio de cooperación. Ministerio de Salud, Instituto Costarricense de Investigación y Enseñanza en Nutrición y Salud, Costa Rica, 12 pp. Descargado de http://www.binasss.sa.cr/Centimic.pdf el julio 2012.

Montero, M., Rojas-Sánchez, F., Socorro, M., Acebedo, A.M., 2007. Dental caries and fluorosis in children consuming water with different fluoride concentrations in Maiquetía. Vargas State, Venezuela. Invest. Clín Journal., 48(1), 5-19.

Murray, J.J., Rugg-Gunn, A.J., Jenkins, G.N., 1991. Fluorides in caries prevention. $3^{\text {rd }}$ edition. Butterworth-Heinemann, Oxford, p. 7-37.

Muzzo, S., Villa, A., 2001. El uso de fluoruro en la prevención de la caries: Sal de mesa y la leche como vehículos sistémicos de fluoruro. Rev. Chilena, 28(1), 149-158.

Ramírez-Puerta, B., Franco, A.M., Ochoa, E., 2006. Fluorosis dental en escolares de 6 a 13 años de Instituciones Públicas de Medellín, Colombia. Rev. de Salud Pública, 11(4), 631-640.

Rosales, S., Maritza, C., 2004. Sistema de Vigilancia para el Programa de Fluoración de la Sal de Consumo Humano en Cuba. Rev. Cub. Salud Pública, 30(4).

Shivarajahankara, Y.M., Shivashankara, A.R., Rao, S.H., Bhat, P.G., 2001. Oxidative stress in children with endemic skeletal fluorosis. Fluoride, 34, 103-107.

Vani, M.L., Reddy, K.P., Bhat, P.G., 2001. Effects of fluoride accumulation on some encimes of brain and gastrocnemius muscle of mice. Fluoride, 33, 17-26.

Wefel, J., Donly, K., 1999. Clínicas odontológicas de Norteamérica. Cariología, 43(4), 779-800.

WHO, 1997. Oral Health Survey. Basics Methods. $4^{\text {th }}$ ed. World Health Organization, Geneva, Suisia, 93 pp. Descargado de http://new.paho.org/hq/dmdocuments/2009/OHstEsurv.pdf el 15 mayo 2012.

Zimmer, S., Jahn, K.R., Barthel, C.R., 2003. Recommendations for the use of fluoride in caries prevention. Oral Health Prev. Dent., 1(1), 45-51. 